\title{
Anion Recognition Via Polarized -N-H Fragments
}

\author{
Dr. Masood Ayoub Kalo, \\ Assistant Professor \& Head, Laboratory of Molecular Materials, Supramolecular Analytical \& Environmental \\ Chemistry, Department of Chemistry, Govt. Degree College (GDC) Shopian (J\&K)-India-192303.
}

makandchem@gmail.com

\begin{abstract}
:
Hydrogen bonding interaction and or proton transfer assets of synthetic molecules in presence of anionic species is pretty fascinating in the field of supramolecular analytical chemistry. Not only amide or urea-based derivatives have appeared in the highlights, rather from last few decades, polarized free amine fragments ($\mathrm{NH}_{2}$ ) have been brought under the study with prompt signaling. In this report, I will be focusing on the basic aspects which trigger free amine group to decode rapid anion recognition not only under organic media, but also under aqueous conditions in diverse environments.
\end{abstract}

\section{Introduction:}

Anion sensing has been an area of enormous interest to research community owing to the widespread existence of anions in diverse matrices like soil, water, air, food and biosystems. ${ }^{1}$ The potential to monitor and chemically characterize such environments is fascinating once we talk of these synthetic tools (receptors) achieved through proper combination of organic synthons. The anion and receptor encounter results in the generation of output signals in the form of color, fluorescence, electrochemical, etc which decipher the qualitative and quantitative estimation of the anions in the sample. ${ }^{2}$ The use of such low cost, easy to handle, minute, quick time response based molecular platforms have proven to provide the potential alternative towards real-time and convenient monitoring of the diverse environments existing in our day to day arena. Among the various categories of receptors by which molecules sense the anions, utilization of organic moieties with polarized $\mathrm{N}-\mathrm{H}$ bonds has been is one the most commonly utilized receptor platform in the supramolecular analytical chemistry. In this direction, the arena has got its shift from amide or urea based fragments to free $-\mathrm{NH}_{2}$ moiety based molecules possessing polarized $\mathrm{N}-\mathrm{H}$ bonds.

\section{Body paragraph}

Organic molecules containing $\mathrm{N}-\mathrm{H}$ bonds wherein hydrogen atom is attached to electron deficient nitrogen center is referred to as polarized $\mathrm{N}-\mathrm{H}$ bond. Such $\mathrm{N}-\mathrm{H}$ bonds are behaved as hydrogen atom donors towards anions in polar aprotic solvents (THF, DMSO, $\mathrm{CH}_{3} \mathrm{CN}, \mathrm{DMF}$, etc.). ${ }^{3}$ Researches with allied fields have utilized various organic moieties which contain such polarized $\mathrm{N}-\mathrm{H}$ bonds for sensing of anions for diverse applications. This is attributed to the fact such an anion receptor encounter has been most of the times reported with beautiful and astonishing visual display in the form of colour and fluorescence. Not only this, research has extended beyond the existing molecular fragments and scientists started designing new molecules as per the target anion. This has been aided by advanced developments in the synthetic organic chemistry. In this molecular engineering several fields of sciences have clubbed together to attains the specific goals and suffice needs of modern day to day life. Some of important fields include transition metal coordination chemistry, anion coordination chemistry, supramolecular chemistry, analytical and bio analytical sciences as well as materials sciences. ${ }^{4}$

For the generation of molecules with polarized $\mathrm{N}-\mathrm{H}$ bonds, a range of molecular fragments have been reported for studying anion recognition chemistry. In this regard, molecules with functionalities like, ureas, amides, thioureas, ammonium, imidazole and imidazolium systems have been reported. ${ }^{5}$ The interactions of anions under the aprotic solvent conditions have resulted in the modulation of electronic properties in the systems which are accompanied by output signals (absorption, emission, electrochemical, etc.). However such 
systems interact with anions via hydrogen bond donation or in extreme cases accompanied by proton transfer from molecule to anion. The events result in the enhancement of electron density on the nitrogen atoms. The concentration of negative charge density on the nitrogen modulated various processes like photo induced electron transfer (PET), Charge transfer (CT), conjugation, etc. ${ }^{6}$ The modulation of such phenomenon produces the basis for locating the anion in any matrix. The changes could be qualitative as well as quantitative. Not only this, the anion signaling events are very fast (within few seconds) and most of the times reversible. Thus signifying the use of these polarized $\mathrm{N}-\mathrm{H}$ bond based molecules for monitoring of such anionic analytes with an ease. Despite all this, certain short comings have been noticed in such class of molecules with $\mathrm{N}-\mathrm{H}$ fragments. Molecular scaffolds suffer from multi-anion interference due to $\mathrm{Y}$-shaped oxoanions through hydrogen bonds. The molecular structure requires tagging of markers due to which cost of the material increases. Besides, some of the events ended up with delayed response. ${ }^{7}$ In order to overcome such shortcomings, recently a new strategy wherein polarized $\mathrm{N}-\mathrm{H}$ bonds have been developed from free $-\mathrm{NH}_{2}$ fragments and suitable for anion recognition. ${ }^{8}$ The realm of such anion polarization was found not only suitable to detect anion in organic solvents but suitable enough to sense anions under aqueous environment. The mechanism of action is attributed to the fact that the free $-\mathrm{NH}_{2}$ group has been set as electron donor (D) at one end of the molecule with respect to some electron acceptor (A) at other end of the molecule. The D and $\mathbf{A}$ fragments were put in strong electronic communication via pi $(\boldsymbol{\pi})$ bonds. This pull-push type of molecular architecture results in the flow of electronic charges away from nitrogen atom of free $-\mathrm{NH}_{2}$, hence $\mathrm{N}-\mathrm{H}$ bond becomes polarized. During the encounter between the anion and the D moiety of molecule which act as receptor site as well, hydrogen bond formation or proton transfer from $-\mathrm{NH}_{2}$ to anion results the enhancement of electron density on the $\mathrm{D}$ site. This promotes a strong intramolecular charge transfer (ICT) across the system hence a prompt and strong readout signaling comes out. Such characteristic features of the newly designed polarized receptor design have overcome most of the shortcomings associated with the earlier receptor based upon amide or urea based fragments.

\section{Conclusions}

In conclusions, I should say that the birth of anion recognition via polarized $\mathrm{N}-\mathrm{H}$ fragments got from natural biological systems like amide bonds in proteins which were embedded by nature and involved in hydrogen bonding interaction. From last five decades researchers have utilized this fact and designed the mimics of this biological architecture in the labs and later utilized for the anon sensing applications in vitro and vivo. Starting from urea or amide based polarized $\mathrm{N}-\mathrm{H}$ bonds; the field has now got extended to free $-\mathrm{NH}_{2}$ fragments. Future looks bright for chemistry and related scientists to sink into this area of this research and expose the futuristic applications.

\section{References}

1. P.A. Gale, Chem. Soc. Rev. 2010, 39, 3746-3771

2. D. Esteban-Gomez, L. Fabbrizzi and M. Licchilli, J. Org. Chem. 2005, 70, 5717-5720.

3. P. A. Gale, E.N.W. Howe and X. Wu, Chem. 2016, 1, 352-422.

4. V. Amendola, D. Esteban-Gomez and L. Fabbrizzi and M. Licchilli, Acc. Chem. Res. 2006, 39, 343-353.

5. M. A. Kaloo and J. Sankar, Analyst. 2013, 238, 4760-4763.

6. M. A. Kaloo and J. Sankar, New. J. Chem. 2014, 48, 923-926.

7. V. Amendola, L. Fabbrizzi and L. Mosca, Chem. Soc. Rev. 2010, 39, 3889.

8. B. Kumar, M. A. Kaloo, A. R. Sekhar and J. Sankar, Dalton. Trans. 2014, 43, 16164-16168. 